\title{
Unilateral absence or extreme hypoplasia of pulmonary veins
}

\author{
H M KINGSTON, R G PATEL, G H WATSON \\ From the Royal Manchester Children's Hospital, Pendlebury, Nr Manchester
}

SUMMARY Three children with recurrent chest symptoms and signs caused by unilateral atresia or absence of the pulmonary veins are described, with a discussion of the seven reported cases of unilateral congenital pulmonary venous obstruction. The condition is rare but should enter into the differential diagnosis of recurrent unilateral pulmonary infection or oedema. Pulmonary arteriogra- $-\infty$ phy is necessary and pulmonary artery wedge angiography is the most informative diagnostic procedure.

Congenital obstruction of the pulmonary veins frequently occurs in patients with total anomalous pulmonary venous drainage, ${ }^{1}$ but is otherwise a rare anomaly. Various forms have been described, involving one to four veins and ranging from localised stenoses to atresia. Bilateral obstruction, unless minimal, leads to pulmonary congestion and oedema and pulmonary arterial hypertension, the clinical course depending on the degree of obstruction. Unilateral obstruction, however, causes less severe symptoms and a clinical picture which may mislead.

We report three cases of unilateral pulmonary venous obstruction, two of which also had congenital cardiac anomalies, and comment on the diagnostic features.

\section{Case reports}

CASE 1

This boy was seen at the age of 14 months shortly after arrival in England from India. His symptoms began at the age of 3 months after which he had recurrent bouts of cough with some wheezing, and also several severe haemoptyses, for which he received a blood transfusion on four occasions. Physical examination disclosed a left leg slightly thinner than the right but no other asymmetry or dysmorphic features; there were a few rales in the left lung. Investigations, including a sweat test and immunoglobulin levels, were normal. A chest $x$-ray film showed a small left lung, with more prominent vascularity on the right. An isotope scan showed normal ventilation and perfusion of the right lung; the left lung showed very little perfusion and some diminished ventilation.

Cardiac catheterisation showed no shunts or intracardiac abnormalities. Oxygen saturations of $6 \$ 0$ to $69 \%$ were found in the superior vena cava, right heart, and right pulmonary artery; that in the lef? pulmonary artery was not significantly different-s $70 \%$. Pulmonary arterial pressures were normal (24f $16 \mathrm{mmHg}$ ), though the left pulmonary arterial wedge pressure was higher than that in the right (14 and 8 $\mathrm{mmHg}$ ). The left atrium could not be entered. Ot angiocardiography an injection into the main pulmo3 nary artery showed a large right pulmonary artery; the left pulmonary artery was relatively small but in absơ. lute terms was only slightly less than the expected size (Fig. 1). The right lung drained normally by large right pulmonary veins into the left atrium, but for? ward flow in the left lung was very slow, and oscilla tory, and arterial branches remained opacified for many seconds after the venous phase on the right. N\& left pulmonary veins could be seen but there was suggestion of small bronchial veins draining the lefe lung (Fig. 2). Aortography was normal and showed ne evidence of sequestration or enlarged bronchiaf. arteries.

Bronchoscopy showed a hyperaemic left bronchial mucosa with increased secretions but the right side was normal.

A left pneumonectomy showed that the left lumg was small, there were some prominent bronchie veins, and two tiny veins ran from the left luns towards the left atrium. On examination of the excised left lung no pulmonary veins could be foundHistological examination showed evidence of som $\Phi$ old and recent haemorrhage in the lung parenchymo? and a very small area of cystic malformation in the lefef 


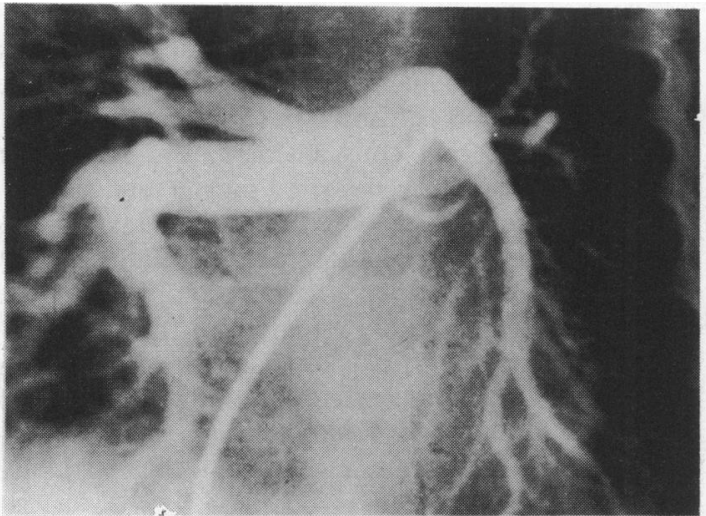

Fig. 1 Case 1: pulmonary arteriogram. Right pulmonary artery large, left slightly small with poor filling of lateral branches.

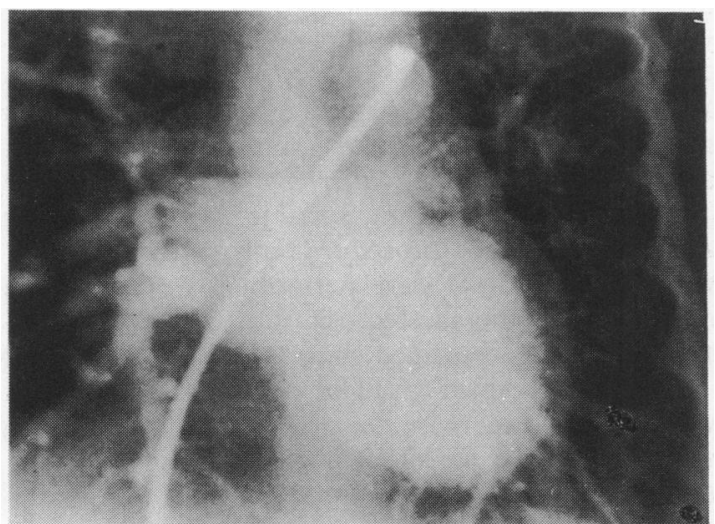

Fig. 2 Case 1: laevophase following Fig. 1. Right pulmonary veins well seen; on the left side some continuing opacification of lower lobe pulmonary artery branches, and some small irregular vessels, but no pulmonary veins visible.

upper lobe, but there was no fibrosis. No pulmonary veins could be seen, but some small veins were present in the peribronchial positions; these veins seemed normal in structure. Some contrast medium which had been injected into the pulmonary artery after removal of the lung could be seen in dilated peribronchial lymphatic vessels.

The child recovered uneventfully and remained well when last heard of at the age of 3 years.

\section{CASE 2}

This boy presented at the age of 1 day with cyanosis. Investigations at that time showed transposition of the great arteries, and anomalous drainage of the right upper lobe pulmonary vein into the upper part of the right superior vena cava. Pressures recorded included: right ventricle $70 / 2$, aorta $70 / 40$, and left ventricle $40 / 2 \mathrm{mmHg}$. Right ventricular and aortic

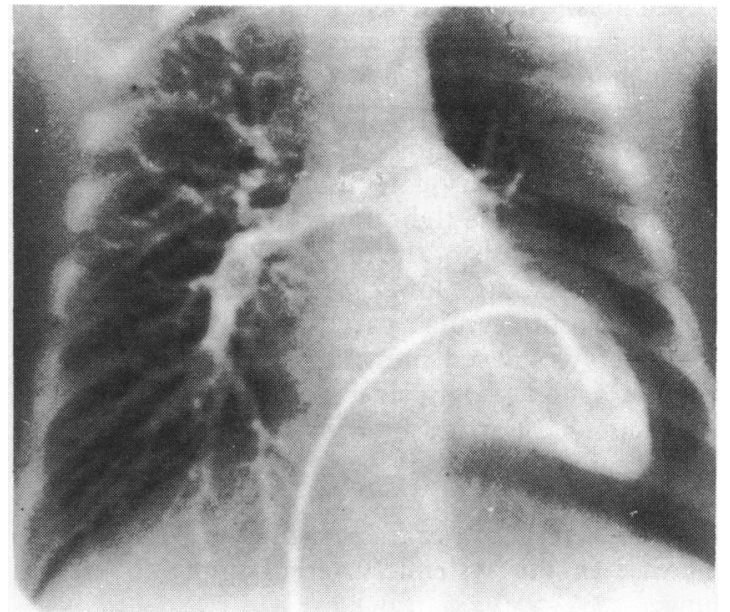

Fig. 3 Case 2: left ventricular angiogram at age 4 months. All medium enters pulmonary artery but most then passes through right lung; left pulmonary artery branches poorly filled.

angiograms excluded a ventricular septal defect; there was a small persistent ductus which later closed spontaneously. A left ventricular angiogram showed contrast medium all passing through an unobstructed outflow tract into unremarkable pulmonary arteries, but the venous return on the left side was obscured by refilling of the left ventricle. The left lung, however, was a little more translucent than the right though of normal şize.

After a balloon septostomy his progress was at first satisfactory, but because of increasing cyanosis he was recatheterised at the age of $\mathbf{4}$ months. After a further balloon septostomy the aortic oxygen saturation was $62 \%$; the pressures had not changed significantly from those recorded at the age of 1 day. A left ventricular angiogram (Fig. 3) showed most of the medium entering the right pulmonary artery, and indeed very little medium passed into the left lung. The right sided pulmonary veins could all be seen but not those on the left, though the reopacified left ventricle obscured the view.

At the age of 14 months a modified Mustard operation was performed, diverting the systemic venous return to the left ventricle and the pulmonary return to the right ventricle. No left sided pulmonary veins could be found. It was not found possible to channel blood from the anomalous right upper lobe pulmonary vein into the pulmonary venous atrium. Nevertheless he made good progress after the operation for about a year but subsequently developed signs of inferior vena caval obstruction with hepatomegaly, and evidence of a right to left shunt with some cyanosis. He also had recurrent episodes of cough and dyspnoea with signs suggesting pulmonary oedema 
predominantly on the left side. On chest $x$-ray films the left lung now looked smaller than the right, and at times there was evidence of pulmonary oedema.

Catheterisation at the age of 3 years (Table 1) showed moderate pulmonary hypertension, with an increased pulmonary flow resulting from the anomalous drainage of the right upper lobe pulmonary vein. There was stenosis of the inferior vena caval pathway and also of the right lower lobe pulmonary vein pathway in its intracardiac part. Left pulmonary artery angiography showed most contrast medium flowing backwards to the right side; medium oscillated in the left pulmonary artery branches and there was little advance. No pulmonary veins could be seen on the left side though there was a suggestion of small bronchial veins on the later frames (Fig. 4). There was no increase in the systemic arterial supply to the left lung.

Table 1 Case 2, cardiac catheterisarion data at age 3 years

\begin{tabular}{lll}
\hline Site & $\begin{array}{l}\text { Oxygen } \\
\text { saturation (\%) }\end{array}$ & $\begin{array}{l}\text { Pressure } \\
(\mathbf{m m} \mathbf{H g})\end{array}$ \\
\hline Innominate veins & 64 & 12 \\
Low superior vena cava & 80 & 10 \\
Left ventricle & 79 & $55 / 4$ \\
Left pulmonary artery & 80 & $52 / 17$ \\
Right pulmonary artery & 77 & $50 / 18$ \\
Right pulmonary artery wedge & 95 & 16 \\
Right lower pulmonary vein & 95 & 16 \\
Pulmonary venous pathway & 94 & 0 \\
$\quad$ (near tricuspid valve) & & $80 / 4$ \\
Right ventricle & 86 & $85 / 55$ \\
Aorta & 88 & \\
\hline
\end{tabular}

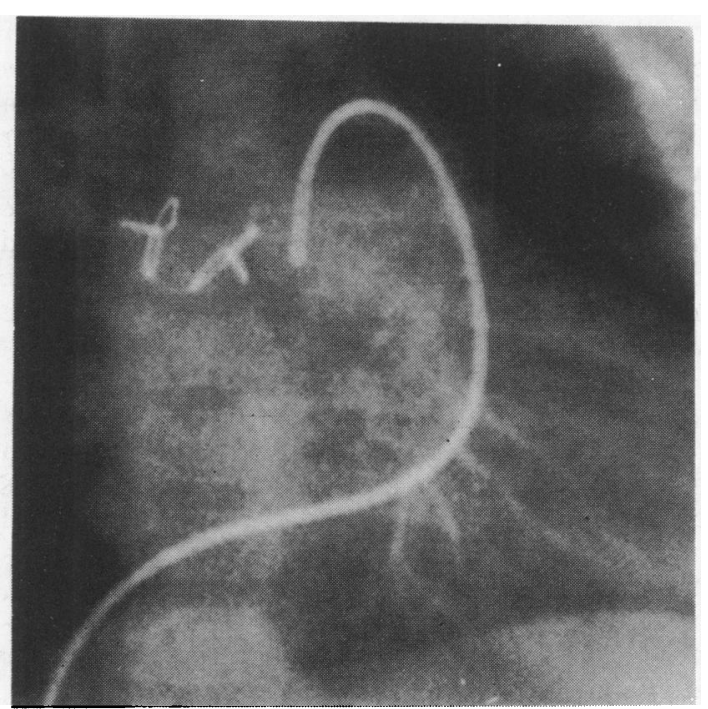

Fig. 4 Case 2: frame taken nine heart beats after injection.

Lefi lower lobe pulmonary artery branches still visible. No left pulmonary veins seen.
Further surgery was undertaken, at which the venous obstructions were relieved and the right upper lobe pulmonary venous blood was redirected into the pulmonary venous atrium, but unfortunately the child did not regain consciousness, and died after renal failure. Necropsy showed a satisfactory anatomical reconstruction. No left sided pulmonary veins could be found. The right lung weighed $520 \mathrm{~g}$, the left only $175 \mathrm{~g}$. Histological examination showed the changes of pulmonary hypertension in the small pulmonary arterioles and areas of bronchopneumonia on both sides. In addition the left lung showed interstitial fibrosis, some areas of infarction, and dilated lymphatic vessels; there were some veins of normal structure but only in peribronchial positions.

\section{CASE 3}

This girl had cyanosis from shortly after birth and was investigated elsewhere at the age of 4 days. On a chest $x$-ray film at this time the lungs were translucent but otherwise unremarkable. Investigations showed isolated pulmonary atresia, with a small right ventricle; the pulmonary arteries were not well seen. After transfer here a right Waterston-Cooley procedure (anastomosis of ascending aorta to right pulmonary artery) and a Brock procedure (enlargement of right ventricular outflow) were performed. A continuous murmur from the anastomosis subsequently became audible and has remained since.

She has remained small in build and moderately cyanosed but otherwise her progress was satisfactory at first. From the age of 18 months she had recurrent episodes of cough and dyspnoea with left sided chest crepitations. Examination showed cyanosis at rest, some clubbing, and bilateral congenital ptosis. Chest $x$-ray film showed variable changes compatible with pulmonary oedema in the left lung, which by the age of 3 years looked smaller than the right.

Two dimensional echocardiography showed the features of isolated pulmonary atresia; the right lower lobe pulmonary vein was seen, but no left sided pulmonary vein could be found.

At catheterisation at the age of 3 years (Table 2) the catheter passed through the right ventricle into the left pulmonary artery but not the right; and through a central atrial septal defect into the left atrium, left ventricle, and right lower lobe pulmonary vein, but not into any left pulmonary veins. There was a variable right ventricular and pulmonary systolic hypertension, with a high end-diastolic right ventricular pressure, and similar tracings in the right ventricle and pulmonary artery. The left pulmonary arterial wedge pressure was very high. There was a rise in oxygen saturation to nearly the aortic level in the right ventricle and left pulmonary artery, and a right to left atrial shunt. 
Table 2 Case 3, cardiac catheterisation data at age 3 years

\begin{tabular}{lll}
\hline Site & $\begin{array}{l}\text { Oxygen } \\
\text { saturation }(\%)\end{array}$ & $\begin{array}{l}\text { Pressure } \\
(\mathrm{mmHg})\end{array}$ \\
\hline Superior vena cava & 62 & - \\
Right atrium & 62 & 7 \\
Right ventricle & 75 & $32 / 8$ to $55 / 10$ \\
Left pulmonary artery & 76 & $34 / 8$ to $55 / 10$ \\
Left pulmonary artery (wedge) & 75 & 50 \\
Right lower pulmonary vein & 95 & 9 \\
Left ventricle & 78 & $96 / 2$ \\
\hline
\end{tabular}

A left atrial angiogram showed no reflux of medium into any of the pulmonary veins and there was washout from the right lung though none from the left. A left ventricular angiogram excluded a ventricular septal defect, all the contrast medium passing into a large ascending aorta; the Waterston anastomosis supplied only the right pulmonary artery. A few small bronchial arteries ran to the left lung. A right ventricular angiogram showed a small poorly functioning cavity; medium advanced into the main pulmonary artery but did not advance into the lungs and flowed back freely into the right ventricle in diastole. An injection into the left pulmonary artery showed some advance of contrast medium into small arterial branches during systole (Fig. 5), but medium then oscillated back in diastole into the main pulmonary artery and right ventricle. As may happen through distortion by the operation the right pulmonary artery was completely obstructed proximal to the Waterston anastomosis. Contrast medium injected through an end-hole catheter in the left pulmonary artery wedge position could be

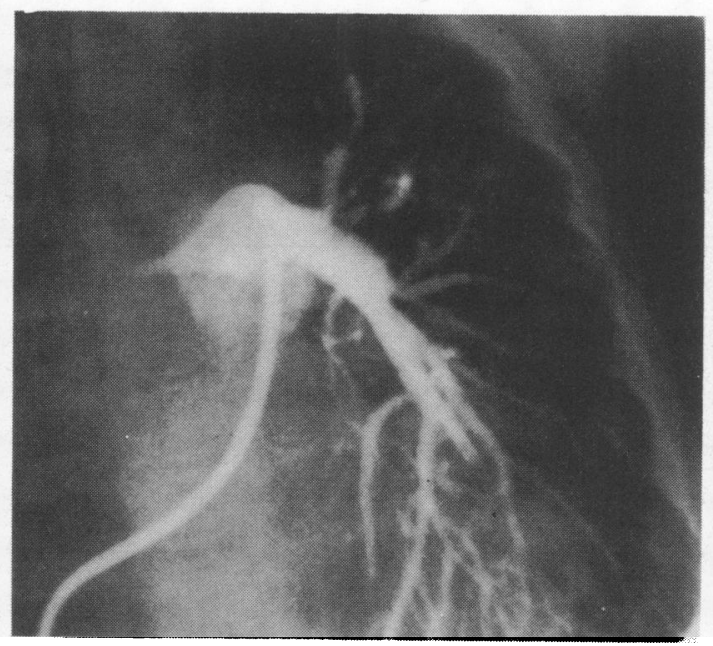

Fig. 5 Case 3: age 3 years. Left pulmonary arteriogram, systole. Opacification of small arteries, of main pulmonary artery, and of proximal part of right pulmonary artery which tapers to occlusion.

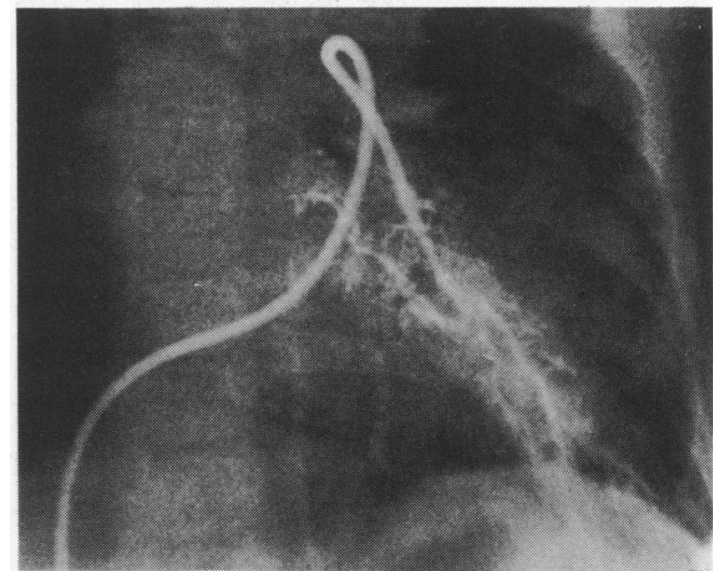

Fig. 6 Case 3: injection through end-hole catheter wedged in lefi lower lobe pulmonary artery. Some hasy capillary filling, and opacification of small distal pulmonary arteries and of irregular vessels running proximally.

seen passing from the small distal pulmonary artery branches into bronchial arteries (Fig. 6) and some of it then flowed back into the pulmonary arteries more proximally (Fig. 7). No pulmonary veins opacifed; later frames (Fig. 8) showed slow filling of what appeared to be small bronchial veins in subpleural positions and also along the left main bronchus to the trachea and even over to the right side.

The little girl remains in much the same health; further operation seemed inadvisable, so no anatomical data are available.

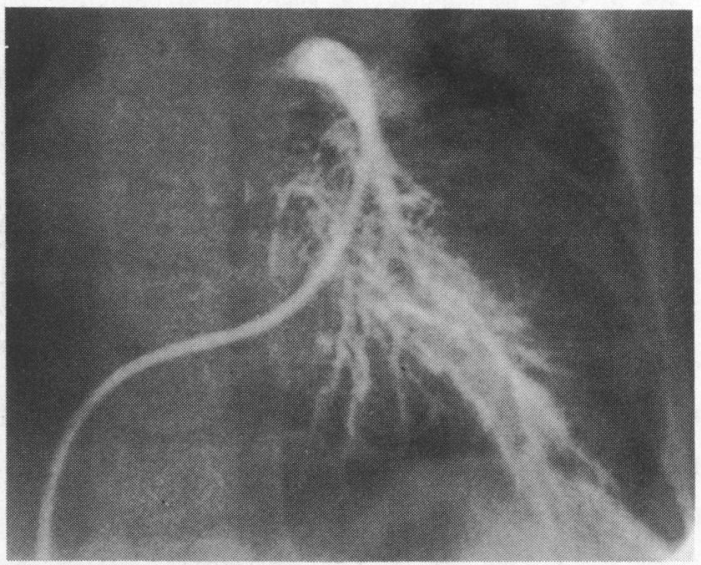

Fig. 7 Case 3: later frame following Fig. 6. Further opacification of distal pulmonary arterial tree and of small irregular vessels more proximally, from some of which contrast medium has entered the main stem of the left pulmonary artery. No pulmonary veins visible. 


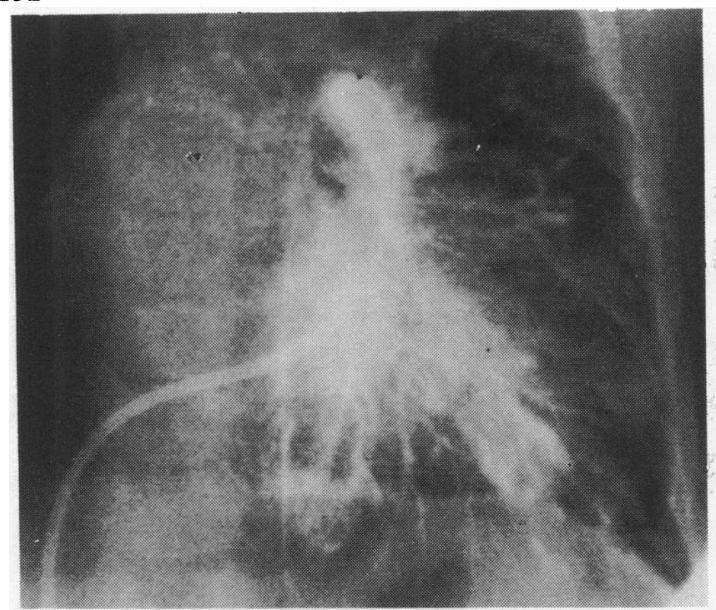

Fig. 8 Case 3: later frame following Fig. 7. Hary capillary blush, and opacification of bronchial veins running along left main bronchus and of subpleural veins, but no pulmonary veins visible.

\section{Discussion}

Each of the three children described had left sided pulmonary venous obstruction. In one the pulmonary veins were absent, in one they were extremely small at operation and could not be found after the lung was removed, and in one case there was no anatomical evidence but good investigatory evidence that the veins were either atretic, extremely small, or absent.

A review of the published reports has disclosed only a few cases of congenital unilateral pulmonary venous obstruction and we have listed these in Table 3, including those with stenosis as well as those with $\stackrel{c}{.}$ atresia, as some of the features are similar. We have $\vec{F}$ excluded that of Samanek et $\mathrm{al}^{9}$ in which the right pulmonary veins were atretic, because congenital obs- $\frac{C}{0}$ truction of the left pulmonary veins, which drained $\bar{\omega}$ into the portal vein, led to severe pulmonary hyper- $\vec{\sigma}$ tension and a different clinical picture. We have found no case reports of unilateral absence of pulmo- $\infty$ nary veins exemplified by our case 2 .

In general, patients have presented with unilateral congestive symptoms and signs or haemoptyses, with $\vec{\sigma}$ $x$-ray changes which have sometimes suggested infection and at times pulmonary venous hypertension.? Gradual relative diminution in size of the affected 0 lung occurred in two of our cases but has not been? commented on previously, though the affected lungs $\vec{\perp}$ appear small in the cases of Bouchard et al. ${ }^{6}$ and $\mathrm{O}$ Nasrallah et al. ${ }^{8}$

Two dimensional echocardiography has not been $\vec{T}$ used in the reported cases. In our case 3 we were able $\mathbb{O}$ to show a large right lower lobe pulmonary vein and? could not detect any left pulmonary vein. We are not yet certain, however, that failure to picture a pulmo- nary vein would be a reliable indication of its absenceos or hypoplasia, and of course demonstration of a nor- mal pulmonary vein would not exclude an intrapulmonary obstruction.

At cardiac catheterisation mild pulmonaryo hypertension has been present in some cases uncomplicated by major abnormalities, but has noo correlated closely with symptoms. Bronchial arteries $\vec{B}$ which commonly communicate with the smalB pulmonary artery branches are presumably?

Table 3 Cases of unilateral pulmonary venous obstruction with unobstructed contralateral veins

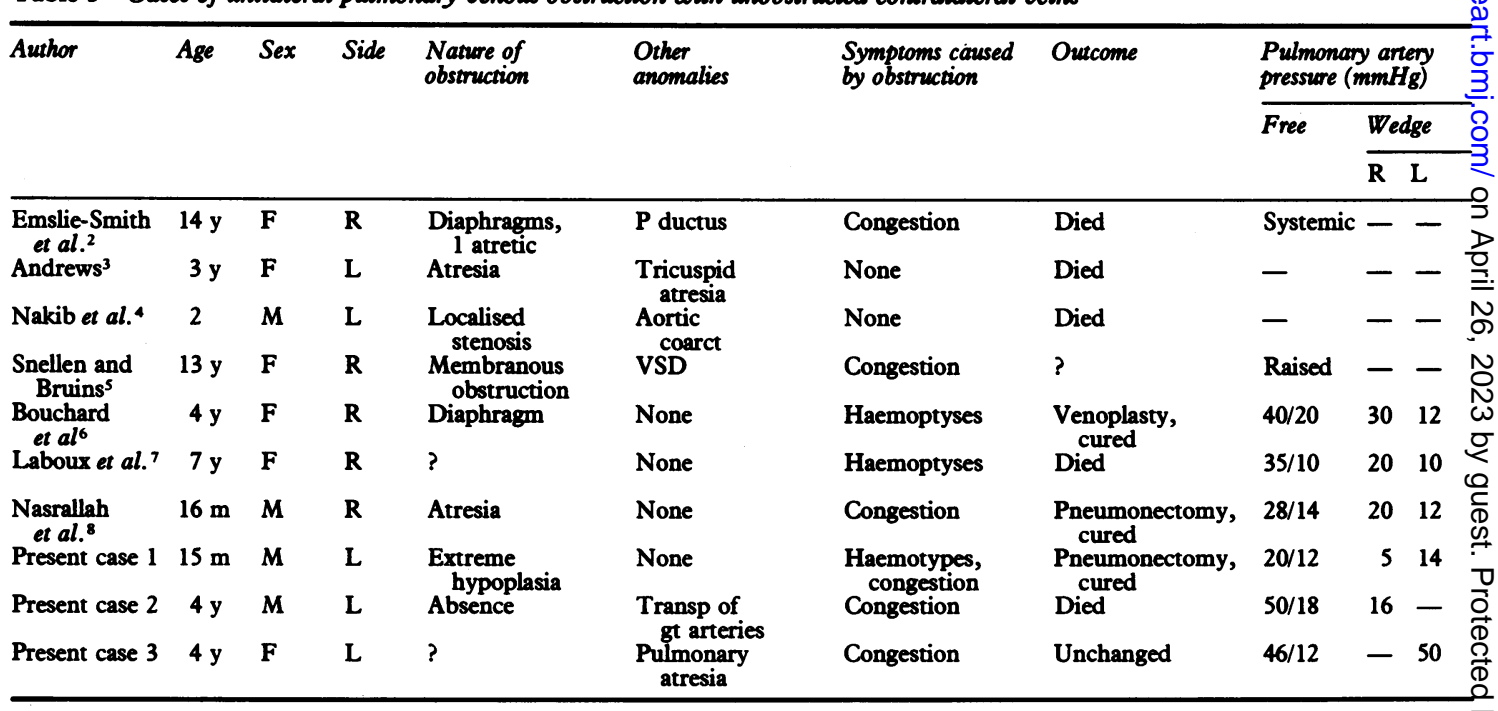


responsible for the raised pulmonary artery wedge pressure and the high pulmonary artery oxygen saturation which may be characteristically found on the affected side.

Pulmonary arteriography on the affected side shows very little forward flow, and contrast medium may oscillate or flow backwards (68 and our three cases). Medium may not enter the affected lung at all, or may remain in it for many seconds. Pulmonary veins are not seen if they are atretic though they may be if merely stenosed. ${ }^{5}$ It may be very difficult, however, to see the pulmonary veins even if present and more information has been obtained from wedge angiography. Pulmonary artery wedge angiography may show filling of small bronchial arteries which may rejoin and opacify the pulmonary arterial tree more proximally (our case 3 ). Subsequently a bronchial venous plexus may be seen, but no true pulmonary veins $\left({ }^{8}\right.$ and our case 3) unless they are merely stenosed as in case described by Bouchard et al. ${ }^{6}$ and also as in a case of acquired stenosis. ${ }^{10}$

Histological changes have included oedema, congestion, dilatation of lymphatic vessels, patchy infarction, probably responsible for some of the radiological shadows, ${ }^{8}$ and fibrosis ${ }^{3}$ (and our case 2). Intrapulmonary veins were sometimes normal (our cases 1 and 2) but sometimes fibrosed ${ }^{8}$ and hypertensive changes were present in the intrapulmonary arteries in some cases. ${ }^{38}$

Treatment would in general be pneumonectomy should symptoms be severe enough, though surgical repair of localised stenosis of veins has been performed successfully in one case. ${ }^{6}$

\section{Conclusion}

Congenital unilateral pulmonary venous obstruction, though rare, should be considered in the differential diagnosis of recurrent bouts of illness suggesting unilateral pneumonia or oedema. Pulmonary arterial wedge angiography is the most helpful single diagnostic measure in determining the nature of the venous obstruction.
We are grateful to $\mathrm{Mr}$ John Dark for the surgical findings and to Dr K V Lodge and Dr M Lendon for the pathological data.

\section{References}

1 Lucas RV, Schmidt RE. In: Moss AJ, Adams FH, Emmanouilides GC, eds. Heart disease in infants, children and adolescents. 2nd ed. Baltimore: Williams and Wilkins, 1978: 458.

2 Emslie-Smith D, Hill IGW, Lowe KG. Unilateral membranious pulmonary venous occlusion, pulmonary hypertension, and patent ductus arteriosus. Br Hear $\mathcal{F}$ 1955; 17: 79-84.

3 Andrews EC Jr. Five cases of an undescribed form of pulmonary interstitial fibrosis caused by obstruction of the pulmonary veins. Bull Fohns Hopkins Hosp 1957; 100: $28-42$.

4 Nakib A, Moller JH, Kanjuh VI, Edwards JE. Anomalies of the pulmonary veins. Am $\mathcal{F}$ Cardiol 1967; 20: 77-90.

5 Snellen HA, Bruins C. In: Watson H, ed. Paediatric cardiology. London: Lloyd-Luke, 1968: 428.

6 Bouchard F, Binet JP, Chetochine F, Conso JF, Langlois J, Pottemain M. Un cas de sténose des veines pulmonaires diagnostiqué et traité avec succès. Arch Mal Coeur 1972: 65: 1143-53.

7 Laboux L, Michaud JL, Cornet E. Atrésie unilatérale des veines pulmonaires. Signes cliniques, hémodynamiques et angiographiques a propos d'une observation. Arch Mal Coeur 1972; 65: 1155-8.

8 Nasrallah AT, Mullins CE, Singer D, Harrison G, Mcnamara DG. Unilateral pulmonary vein atresia: diagnosis and treatment. Am F Cardiol 1957; 36: 969-73.

9 Šamánek M, Túma S, Benešová D, Povyšilová V, Pražsky F, Capova E. Atresia of right pulmonary veins and anomalous left pulmonary venous drainage into portal circulation. Thorax 1974; 29: 446-50.

10 Bini RM, Bargeron LM Jr. Visualisation of pulmonary vein obstruction by pulmonary artery wedge injection. Pediatr Cardiol 1982; 2: 161-2.

Requests for reprints to Dr G H Watson, Royal Manchester Children's Hospital, Pendlebury, Near Manchester M27 1HA. 